\title{
Histologic changes associated with the use of fibrinogen- and thrombin-impregnated collagen in the prevention of pulmonary air leakage
}

Norifumi Tsubokawa, MD, ${ }^{\mathrm{a}}$ Yoshihiro Miyata, MD, PhD, ${ }^{\mathrm{a}}$ Takahiro Mimae, MD, PhD, ${ }^{\mathrm{a}}$

Shinsuke Sasada, MD, PhD, ${ }^{a}$ Tomoharu Yoshiya, MD, ${ }^{a}$ Takeshi Mimura, MD, PhD, ${ }^{a}$

Koji Arihiro, MD, $\mathrm{PhD},{ }^{\mathrm{b}}$ and Morihito Okada, $\mathrm{MD}, \mathrm{PhD}^{\mathrm{a}}$

\section{ABSTRACT}

Objective: Although fibrinogen- and thrombin-impregnated collagen (TachoSil; Takeda GmbH, Linz, Austria) can be applied to prevent air leakage, the impact of its use on lung healing is unknown. Therefore, we histologically evaluated the long-term healing process associated with the use of TachoSil to prevent air leakage in a canine model.

Methods: Via left thoracotomy, visceral pleural defects of $10 \times 10 \mathrm{~mm}$ were created on each lung lobe of female beagles. After air leakage was confirmed, each pleural defect was covered with TachoSil. The repair sites were histologically evaluated on postoperative days $0,4,7,14,28$, and 56 .

Results: All animals survived, and none developed pneumothorax. Histologically, inflammatory cells infiltrated the TachoSil from the pleural defect, and pleural mesothelium comprised the regenerated outermost layer of the TachoSil soon after the surgery. Inflammatory cells, myofibroblasts, and neovascular vessels subsequently spread over the entire TachoSil. The number of inflammatory cells decreased, and myofibroblast and neovascular vessels replaced the entire TachoSil. In addition, the elastic layer started to regenerate from both edges and completely repaired the pleural defect. The lung parenchyma around the pleural defects was not influenced throughout the observational period, because these healing processes occurred only inside the TachoSil.

Conclusions: TachoSil provided a mechanical scaffold on which healing could proceed, followed by biodegradation over the long term. TachoSil safely repaired the pleural defects without affecting lung parenchyma. (J Thorac Cardiovasc Surg 2015;149:982-8)

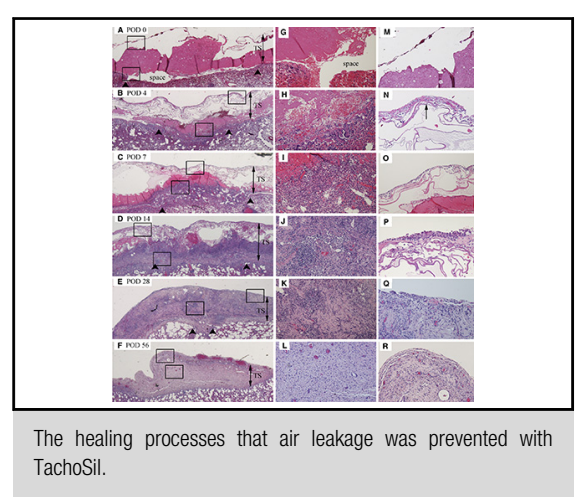

Central Message

TachoSil safely prevents air leakage without affecting lung parenchyma. It provided a mechanical scaffold on which healing could proceed, followed by biodegradation over the long term.

\section{Perspective}

$\overline{\text { Although TachoSil can be applied to prevent air leakage, the }}$ impact of its use on lung healing is unknown. This study histologically showed the long-term healing processes associated with TachoSil, which is used to prevent air leakage. TachoSil provided a mechanical scaffold on which healing could proceed, with subsequent replacement by myofibroblasts and neovascular vessels. TachoSil safely prevented air leakage without negatively affecting the lung parenchyma and without leaving remaining foreign material.

See Editorial Commentary page 989.

See Editorial page 960
Postoperative air leakage is a frequent complication of pulmonary resection. ${ }^{1,2}$ Persistent air leakage has negative consequences on morbidity, culminating in a lengthy hospitalization with negative economic effects and delayed adjuvant treatment. ${ }^{3-7}$ Therefore, a safe and effective procedure is required to prevent and control air leakage.

From the Departments of Surgical Oncology ${ }^{\mathrm{a}}$ and Pathology, ${ }^{\mathrm{b}}$ Hiroshima University, Hiroshima, Japan.

Received for publication Sept 8, 2014; revisions received Dec 21, 2014; accepted for publication Dec 25, 2014; available ahead of print Jan 29, 2015.

Address for reprints: Morihito Okada, MD, PhD, Department of Surgical Oncology, Hiroshima University, 1-2-3 Kasumi, Minami-ku, Hiroshima 734-8551, Japan (E-mail: morihito1217@hiroshima-u.ac.jp).

$0022-5223 / \$ 36.00$

Copyright ( $) 2015$ by The American Association for Thoracic Surgery

http://dx.doi.org/10.1016/j.jtcvs.2014.12.058
TachoSil (Takeda GmbH, Linz, Austria) has been successfully used to reduce the prevalence of the complication of air leakage after pulmonary resection. ${ }^{2,3,8-11}$ However, the impact of TachoSil use on lung healing is unknown. Therefore, we histologically evaluated the long-term healing processes associated with the use of TachoSil to prevent air leakage in a canine model.

\section{MATERIALS AND METHODS \\ Animals and Initial Operation}

Female beagles (Kitayama Labes Co Ltd, Nagano, Japan) were preanesthetized with ketamine $(10 \mathrm{mg} / \mathrm{kg})$ and atropine sulfate $(0.5 \mathrm{mg})$, intubated under general anesthesia ( $5 \mathrm{mg} / \mathrm{kg}$ propofol intravenously; $1 \mathrm{mg} / \mathrm{kg}$ succinylcholine intravenously), and placed under controlled ventilation. Cefazolin $(1 \mathrm{~g})$ was intraoperatively administered. Anesthesia was maintained by periodic injections of propofol and succinylcholine. 


\section{Abbreviations and Acronyms}

$\alpha$-SMA $=\alpha$-smooth muscle actin

$\mathrm{POD}=$ postoperative day

\section{Surgical Procedure}

Animals were placed in the right lateral position for a left thoracotomy. Visceral pleural defects of $10 \times 10 \mathrm{~mm}$ were created on the side of the chest wall, the mediastxinal side, and the interlobar side by sharp dissection. We dissected lung parenchyma to the depths confirming bleeding and air leakage from the created pleural defect. Constriction was applied if needed, but cautery was not. TachoSil was applied to cover each pleural defect with an overlap of more than $5 \mathrm{~mm}$ and held against the lung tissue for 5 minutes (Figure 1). We macroscopically confirmed that TachoSil firmly attached to the defect. No visible air leakage was confirmed. The chest wall was closed after air evacuation by chest tube. After no air leakage was observed, the chest tube was removed before the dogs were extubated. The animals were returned to their cages after being injected intramuscularly with ketoprofen $(1 \mathrm{mg} / \mathrm{kg})$.

Animals were euthanized by a pentobarbital overdose on postoperative days (PODs) $0,4,7,14,28$, and 56. Six dogs were used; 1 was killed on each POD. Three or 4 pleural defects were created in 1 dog for each time point. Thoracotomy in a different intercostal space was performed to macroscopically evaluate postoperative pleural adhesions. The left lung and the surrounding adherent tissue were harvested for each lobe except for the case processed on POD 14, in which en bloc resection was performed because of interlobar adhesion.

\section{Histologic Findings}

Specimens were fixed in $10 \%$ buffered formalin, and the pleura covered by TachoSil was serially cut into $5 \mu \mathrm{m}$-thick slices. Because TachoSil was not detected by macroscopic findings on POD 56, the entire lobe was serially cut into $5 \mu \mathrm{m}$-thick sections and then all paraffin-embedded sections were evaluated by hematoxylin-eosin staining. Pleural defects and the proliferation of myofibroblasts and neovascular vessels were respectively assessed by staining the sections with Elastica van Gieson, $\alpha$-smooth muscle actin ( $\alpha$-SMA), and CD31. The expression levels of each cell were defined as follows: $1+=$ mean low levels, $2+=$ intermediate levels, and $3+=$ high levels of inflammatory cells, myofibroblasts, and neovascular vessels. The Ethics Committee of Hiroshima University approved the study protocol, and laboratory animal care proceeded according to the guidelines for laboratory animal care. ${ }^{12}$

\section{RESULTS}

\section{Macroscopic Findings}

A total of 20 TachoSil patches (3-4 per dog) were applied to pleural defects in the dogs. All dogs survived without severe complications throughout the observation period. Further, all TachoSil patches were attached to the lung parenchymal surface. Extensive inflammation or pneumothorax was not observed at the time of sacrifice (Table 1). Two adhesions $(10 \%)$ were found in the pleura covered by TachoSil: 1 each in the interlobar and mediastinal pleura on PODs 7 and 14, respectively. TachoSil was macroscopically detectable until POD 28 and only microscopically detectable on POD 56.

\section{Microscopic Findings}

Pleural defects were confirmed as spaces caused by air leakage on POD 0 (Figure 2, $A$ ) and as the loss of the elastic layer on Elastica van Gieson-stained sections (Figure 3).
The time-dependent changes of each of the cells are summarized in Table 1. The mesothelium had formed and covered the outermost layer of the TachoSil after POD 4 (Figure 2, N). Inflammatory cells such as lymphocytes and neutrophils initially infiltrated the space, which became filled with these cells and blood cells on POD 4 (Figure 2, B). Subsequently, inflammatory cells spread widely to the TachoSil from POD 7 (Figure 2,C) to 28 (Figure 2,E), but the cell numbers had decreased by POD 56 (Figure 2, F). However, the myofibroblasts and neovascular vessels exhibited histologic characteristics that differed from those of the inflammatory cells. The myofibroblasts and neovascular vessels formed in the TachoSil around the pleural defects on POD 7 (Figure 4, C and $I$ ), which was after the inflammatory cells were first observed, and increased progressively from POD 7 to 28 (Figure 4, $E$ and $K$ ). Finally, the myofibroblasts and neovascular vessels remained in the TachoSil, occupying the entire TachoSil on POD 56 (Figure 4, $F$ and $L$ ). In addition, by POD 14, the pleural defects were confirmed to be loss of the elastic layer (Figure 3, $A-D$ ). However, elastic layer regeneration originated from both edges, and pleural defects started to decrease on POD 28 (Figure 3,E). The pleural defect was completely repaired by POD 56 (Figure 3,F). Because these healing processes occurred only inside the TachoSil, the lung parenchyma around the pleural defects was not influenced throughout the observational period.

\section{DISCUSSION}

This study histologically demonstrated the long-term healing processes associated with TachoSil, which is used to prevent air leakage. TachoSil safely repaired pleural defects to prevent air leaks without negatively affecting the lung parenchyma and without leaving remaining foreign material. TachoSil provided a mechanical scaffold on which healing could proceed, followed by biodegradation over the long term.

Because $90 \%$ of air leaks resolve by POD $7,{ }^{13}$ we included 2 observation end points in this study: prevention of air leakage in the early postoperative period (PODs 0,4 , and 7) and assessment of possible adverse effects in the late period (PODs 14, 28, and 56). The pleural spaces responsible for air leakage were replaced with inflammatory cells and blood cells on POD 4, and these changes became pronounced on POD 7. In addition, the pleural area around the defects adhered to the TachoSil, and these histologic changes were responsible for prevention of air leakage. A previous study ${ }^{14}$ demonstrated the histologic changes associated with the use of fibrin glue-coated collagen fleece to repair pleural defects until POD 7 , and these findings were consistent with our results. However, the changes in the TachoSil and their long-term effects on the lung parenchyma have remained unclear. The present study showed 


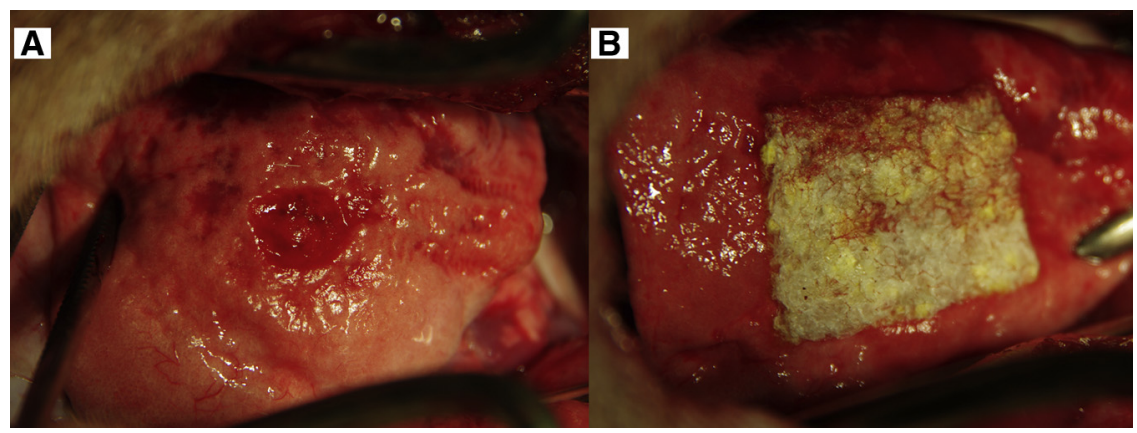

FIGURE 1. Pleural defects and TachoSil (Takeda GmbH, Linz, Austria) cover. Pleural defects of $10 \times 10 \mathrm{~mm}$ were created by sharp dissection (A). TachoSil covers pleural defect with overlap greater than $5 \mathrm{~mm}$ (B). TachoSil collagen patch coated with human fibrinogen and thrombin.

that the TachoSil was completely biodegraded over the long term without negatively affecting the lung parenchyma. After inflammatory cells infiltrated the pleural defects, myofibroblasts and neovascular vessels formed and spread over the entire TachoSil with the inflammatory cells. Finally, the number of inflammatory cells decreased, and the myofibroblasts and neovascular vessels replaced the TachoSil. The TachoSil did not remain as a foreign material. Adverse effects on the lung parenchyma were not found because these healing processes occurred only inside the TachoSil.

In addition, few adhesions were detected in the area covered by the TachoSil, and several possibilities can be proposed to explain this finding. First, although an inflammatory reaction occurred within the TachoSil, it did not occur beyond the TachoSil. Second, the outermost layer of the TachoSil became covered with 1 layer of mesothelium from POD 4, and a rapidly developing mesothelium could prevent adhesions. A relationship between TachoSil and adhesions has been identified in intra-abdominal, ${ }^{15}$ pericardial, ${ }^{16}$ and gynecologic ${ }^{17}$ regions. To our knowledge, pleural adhesions have been prevented only in a few studies in rat models. ${ }^{18,19}$ One study in a rat model ${ }^{19}$ showed that the pleural defects that were healed by the use of hemostatic fleece became covered by regular mesothelium, and the fleece prevented the development of pleural adhesions. Further, hemostatic fleece promoted rapid mesothelial recovery and reduced plasminogen activator inhibitor-1 levels, leading to the biochemical inhibition of adhesion formation. ${ }^{18}$

The myofibroblasts and elastic layer comprised an important part of the healing process. Myofibroblasts regulate the remodeling of connective tissue by combining the extracellular matrix-synthesizing features of fibroblasts with the cytoskeletal characteristics of contractile smooth muscle cells. ${ }^{20}$ Myofibroblasts that are characterized by the expression of $\alpha$-SMA represent key players in the physiologic reconstruction of connective tissue after injury and in generating the pathologic tissue deformations that characterize fibrosis. $^{20-24}$ Our findings demonstrate that similar processes occurred during the healing of pleural defects by TachoSil because the $\alpha$-SMA-positive layer, which indicates the presence of myofibroblasts, gradually thickened from POD 7 to 56 inside the TachoSil. Excessive and persistent myofibroblast activities transform beneficial tissue repair into the detrimental tissue deformities characteristic of organ fibrosis. ${ }^{20}$ However, the myofibroblasts acted only inside the TachoSil, and not in the lung parenchyma, without showing evidence of excessive activity. The elastic layer was also involved in the healing procedure. This study demonstrated that the elastic layer regenerated from both edges on POD 28, with the pleural defects disappearing by POD 56 . We previously reported that the processes associated with the TachoComb (Takeda $\mathrm{GmbH})$ during healing of an injured pulmonary artery

TABLE 1. Surgical and postoperative pathologic findings of TachoSil (Takeda GmbH, Linz, Austria)

\begin{tabular}{|c|c|c|c|c|c|c|}
\hline POD & $\mathbf{0}$ & 4 & 7 & 14 & 28 & 56 \\
\hline No. of TachoSil patches for $1 \mathrm{dog}$ & $\mathbf{n}=\mathbf{3}$ & $\mathbf{n}=\mathbf{4}$ & $\mathbf{n}=\mathbf{3}$ & $\mathbf{n}=\mathbf{4}$ & $\mathbf{n}=\mathbf{3}$ & $\mathbf{n}=\mathbf{3}$ \\
\hline Adhesion & 0 & 0 & 1 & 1 & 0 & 0 \\
\hline Macroscopic confirmation & + & + & + & + & + & - \\
\hline Regeneration of mesothelium & - & + & + & + & + & + \\
\hline Inflammatory cells & - & + & $3+$ & $3+$ & $2+$ & - \\
\hline Myofibroblasts & - & + & + & $2+$ & $3+$ & $3+$ \\
\hline Neovascular vessels & - & + & $2+$ & $2+$ & $2+$ & $2+$ \\
\hline Regeneration of elastic layers & - & - & - & - & + & + \\
\hline
\end{tabular}

$P O D$, Postoperative day. 

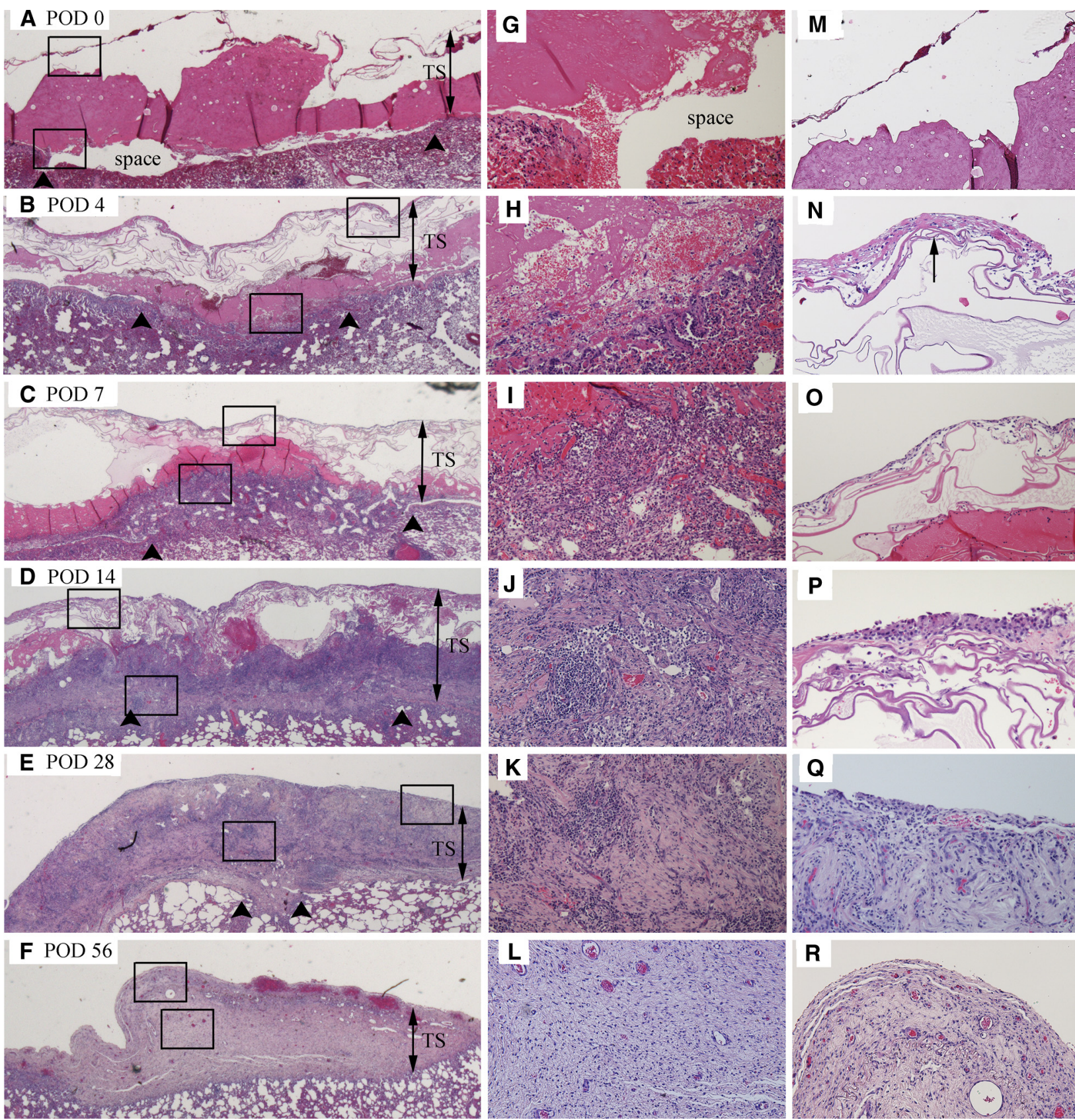

FIGURE 2. Postoperative hematoxylin-eosin staining. Postoperative hematoxylin-eosin staining visualized using loupe (A-F). Around pleural defects (G-L), magnification, $\times 100$. Outermost layer of TachoSil (M-R), magnification, $\times 100$. Arrowheads shows both edges of elastic layer (confirmed by Elastica van Gieson staining in Figure 3). Inflammatory infiltration was noted immediately on POD 4, increased progressively from POD 4 to 28 , and was resolved by POD 56. In addition, mesothelium (arrow) covers the outermost layer of TachoSil on POD 4 (N). TachoSil collagen patch coated with human fibrinogen and thrombin. POD, Postoperative day; TS, TachoSil.

started to extend into the defect between the endothelial layer and the TachoComb from both edges. ${ }^{25}$ The present findings were similar, although the reason for regeneration of the elastic layer remains unknown.

\section{Study Limitations}

First, the canine model differed from the condition that is observed in a clinical setting. Clinically, postoperative air leakage often occurs in patients with emphysematous or fibrotic patterns, whereas the lung was normal in the canine model used in this study. In addition, the pleural defects were small and created without removal of the lung. Thus, the pleural defects may have healed relatively quickly with complete lung reexpansion and apposition of the lung to the surrounding tissues. Further studies are required in a setting that is more similar to that observed 
A POD 0

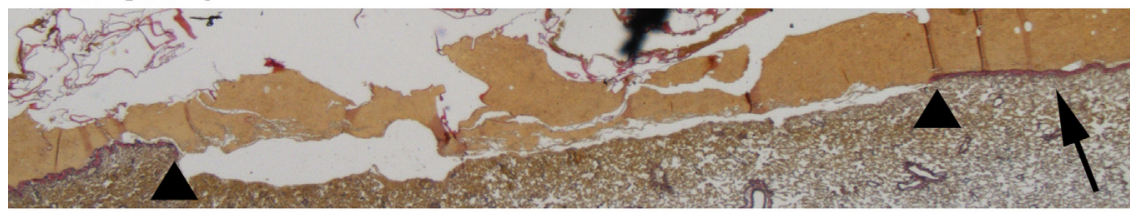

B POD 4

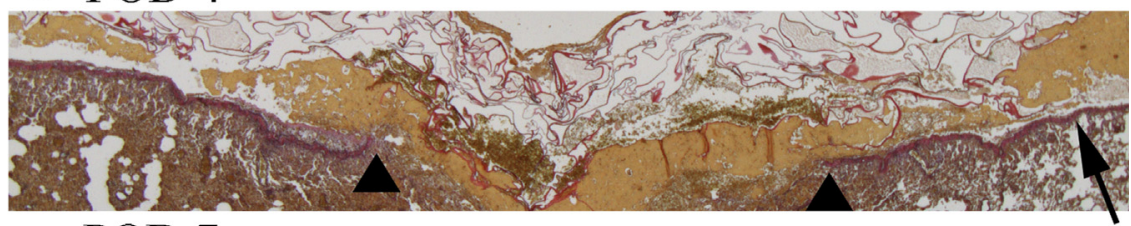

c $\mathrm{POD} 7$

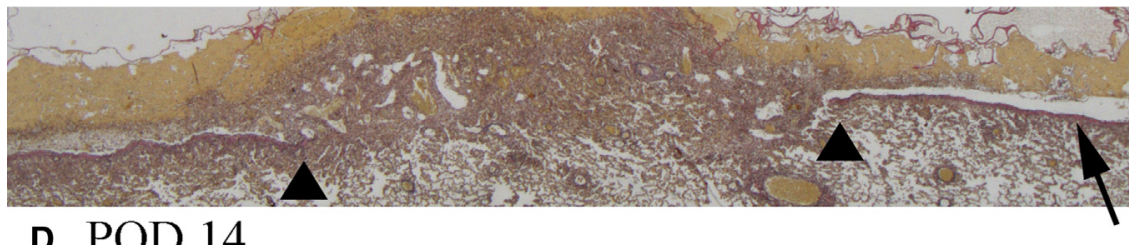

D POD 14

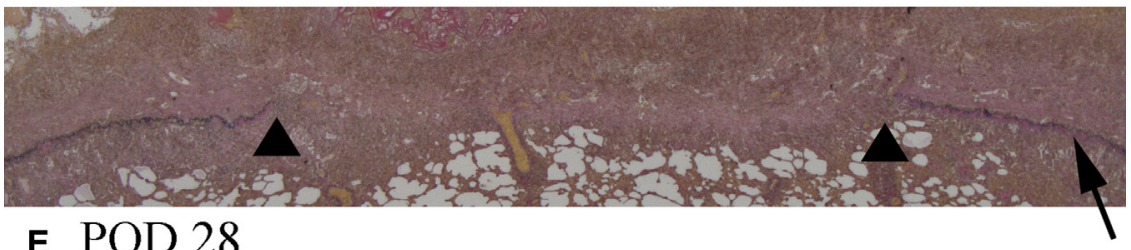

E POD 28

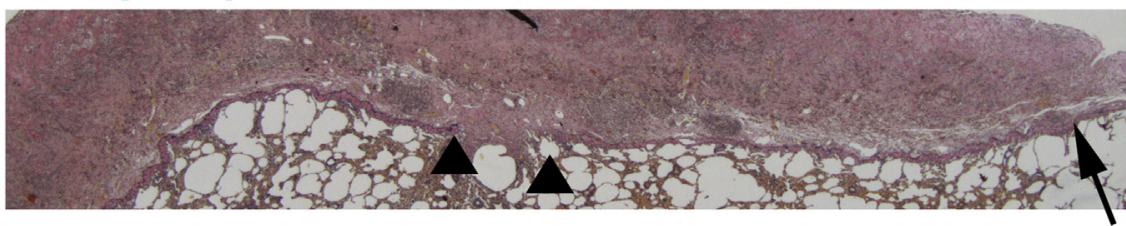

F POD 56

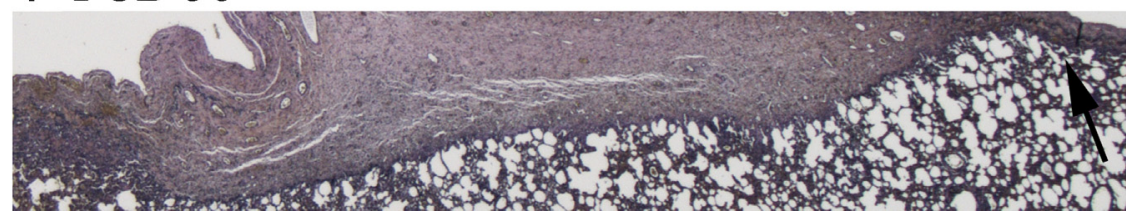

FIGURE 3. Findings of Elastica van Gieson staining. Sections stained with Elastica van Gieson visualized using a loupe (A-F). Arrow and arrowheads show elastic layer and both edges of it, respectively. Elastic layer started to regenerate from both edges on POD 28 (E) and exhibited repair by POD 56 (F). $P O D$, Postoperative day.

clinically. Second, this study did not include a control group, and we assessed only a single animal at each time point. However, although the results in this study were not adequate for performing a quantitative analysis, similar results were obtained in 3 to 4 tissues per time point. Given this finding, we presumed that including additional animals would not greatly change the overall results, so we minimized the number of dogs used from the standpoint of animal welfare. These limitations should be considered when interpreting the results and conclusions of this study.

\section{CONCLUSIONS}

Our study histologically demonstrated the long-term healing processes associated with TachoSil, which is used to prevent air leakage. TachoSil safely repaired pleural defects without negatively affecting the lung parenchyma and without leaving remaining foreign material.

\section{Conflict of Interest Statement}

Authors have nothing to disclose with regard to commercial support. 

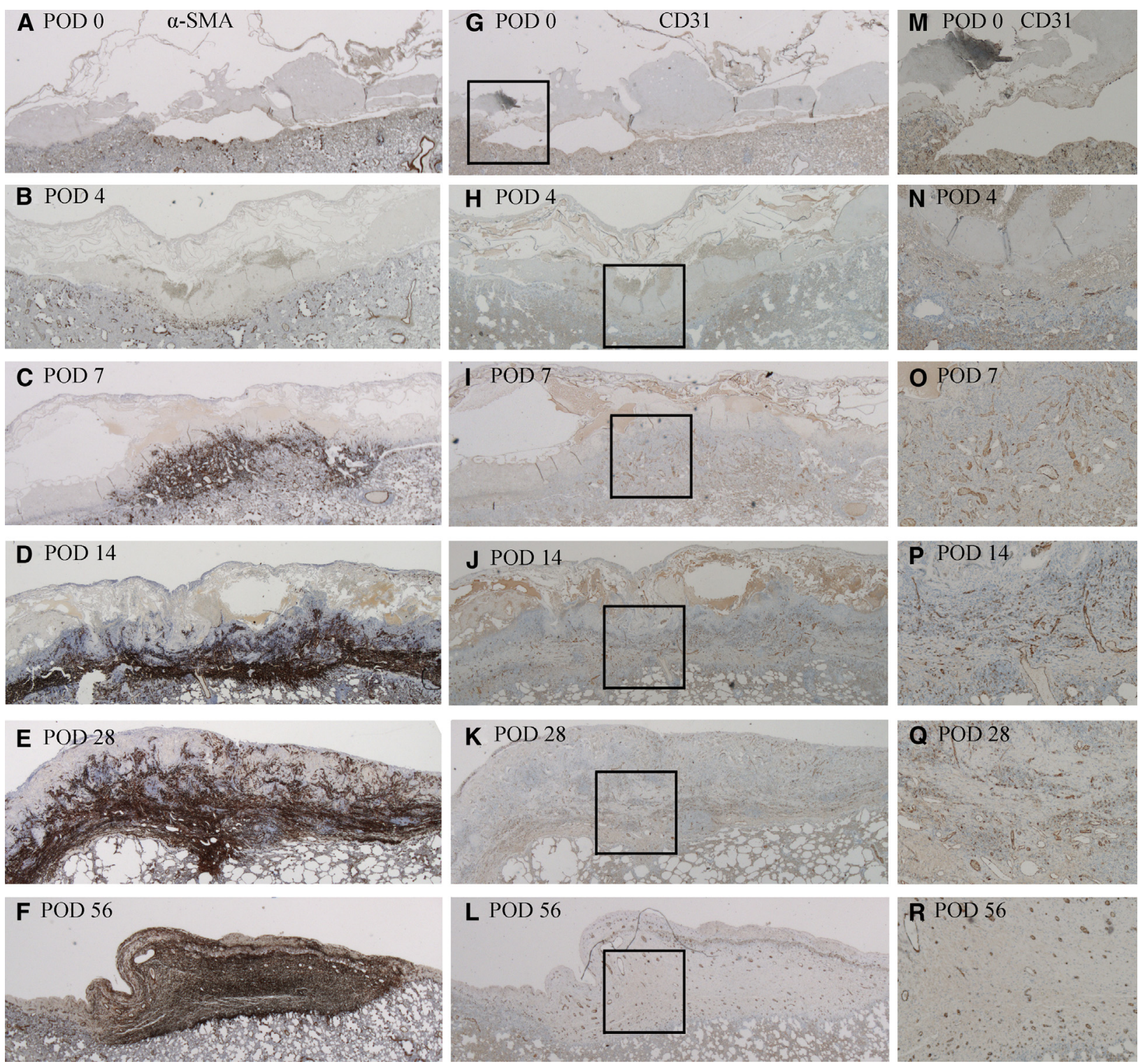

FIGURE 4. Staining of myofibroblasts and neovascular vessels. Sections stained with $\alpha$-SMA (A-F) and CD31 visualized using a loupe (G-L) and CD31 stain at $\times 100$ magnification (M-R). Myofibroblasts and neovascular vessels have formed and widely spread throughout TachoSil from POD 7 to 28 , occupying the entire TachoSil on POD 56. TachoSil collagen patch coated with human fibrinogen and thrombin. $\alpha$-SMA, $\alpha$-Smooth muscle actin; POD, postoperative day.

\section{References}

1. Wain JC, Kaiser LR, Johnstone DW, Yang SC, Wright CD, Friedberg JS, et al. Trial of a novel synthetic sealant in preventing air leaks after lung resection. Ann Thorac Surg. 2001;71:1623-9.

2. Anegg U, Lindenmann J, Matzi V, Smolle J, Maier A, Smolle-Juttner F. Efficiency of fleece-bound sealing (TachoSil) of air leaks in lung surgery: a prospective randomised trial. Eur J Cardiothorac Surg. 2007;31:198-202.

3. Droghetti A, Schiavini A, Muriana P, Folloni A, Picarone M, Bonadiman C, et al. A prospective randomized trial comparing completion technique of fissures for lobectomy: stapler versus precision dissection and sealant. J Thorac Cardiovasc Surg. 2008;136:383-91.

4. Cerfolio RJ, Tummala RP, Holman WL, Zorn GL, Kirklin JK, McGiffin DC, et al. A prospective algorithm for the management of air leaks after pulmonary resection. Ann Thorac Surg. 1998;66:1726-31.

5. Stephan F, Boucheseiche S, Hollande J, Flahault A, Cheffi A, Bazelly B, et al. Pulmonary complications following lung resection: a comprehen- sive analysis of incidence and possible risk factors. Chest. 2000;118: 1263-70.

6. Brunelli A, Monteverde M, Borri A, Salati M, Marasco RD, Fianchini A. Predictors of prolonged air leak after pulmonary lobectomy. Ann Thorac Surg. 2004;77: 1205-10.

7. Venuta F, Rendina EA, De Giacomo T, Flaishman I, Guarino E, Ciccone AM, et al. Technique to reduce air leaks after pulmonary lobectomy. Eur J Cardiothorac Surg. 1998;13:361-4.

8. Filosso PL, Ruffini E, Sandri A, Lausi PO, Giobbe R, Oliaro A. Efficacy and safety of human fibrinogen-thrombin patch $\left(\right.$ TachoSil $\left.{ }^{\circledR}\right)$ in the treatment of postoperative air leakage in patients submitted to redo surgery for lung malignancies: a randomized trial. Interact Cardiovasc Thorac Surg. 2013;16:661-6.

9. Lang G, Csekeo A, Stamatis G, Lampl L, Hagman L, Marta GM, et al. Efficacy and safety of topical application of human fibrinogen/thrombin-coated collagen patch (TachoComb) for treatment of air leakage after standard lobectomy. Eur J Cardiothorac Surg. 2004;25:160-6. 
10. Rena O, Papalia E, Mineo TC, Massera F, Pirondini E, Turello D, et al. Air-leak management after upper lobectomy in patients with fused fissure and chronic obstructive pulmonary disease: a pilot trial comparing sealant and standard treatment. Interact Cardiovasc Thorac Surg. 2009;9:973-7.

11. Marta GM, Facciolo F, Ladegaard L, Dienemann H, Csekeo A, Rea F, et al. Efficacy and safety of TachoSil ${ }^{\circledR}$ versus standard treatment of air leakage after pulmonary lobectomy. Eur J Cardiothorac Surg. 2010;38:683-9.

12. Guide for the Care and Use of Laboratory Animals. Washington DC: National Academy of Sciences; 1996.

13. Okereke I, Murthy SC, Alster JM, Blackstone EH, Rice TW. Characterization and importance of air leak after lobectomy. Ann Thorac Surg. 2005;79:1167-73.

14. Matsutani N, Ozeki Y. Pleural defect repair with an overlapping method using fibrin glue-coated collagen fleece. Surg Today. 2011;41:1234-7.

15. Schneider A, Bennek J, Olsen KO, Weiss J, Schmidt W, Rolle U. Experimental study evaluating the effect of a barrier method on postoperative intraabdominal adhesions. Dig Dis Sci. 2006;51:566-70.

16. Kuschel TJ, Gruszka A, Hermanns-Sachweh B, Sachweh B, Elyakoubi J, Sachweh JS, et al. Prevention of postoperative pericardial adhesions with TachoSil. Ann Thorac Surg. 2013;95:183-8.

17. Dickneite G, Rolle U, Rosenthal D. Prevention of gynaecological adhesions using haemostatic fleece in a rabbit model. J Int Med Res. 2006;34:505-13.
18. Komatsu K, Fujii A, Higami T. Haemostatic fleece (TachoComb) to prevent intrapleural adhesions after thoracotomy: a rat model. Thorac Cardiovasc Surg. 2007 55:385-90

19. Getman V, Devyatko E, Wolner E, Aharinejad S, Mueller MR. Fleece bound sealing prevents pleural adhesions. Interact Cardiovasc Thorac Surg. 2006;5:243-6.

20. Hinz B, Phan SH, Thannickal VJ, Prunotto M, Desmouliere A, Varga J, et al. Recent developments in myofibroblast biology: paradigms for connective tissue remodeling. Am J Pathol. 2012;180:1340-55.

21. Hinz B, Phan SH, Thannickal VJ, Galli A, Bochaton-Piallat ML, Gabbiani G. The myofibroblast: one function, multiple origins. Am J Pathol. 2007;170: 1807-16.

22. Gabbiani G. The myofibroblast in wound healing and fibrocontractive diseases. $J$ Pathol. 2003;200:500-3.

23. Desmouliere A, Chaponnier C, Gabbiani G. Tissue repair, contraction, and the myofibroblast. Wound Repair Regen. 2005;13:7-12.

24. Tomasek JJ, Gabbiani G, Hinz B, Chaponnier C, Brown RA. Myofibroblasts and mechano-regulation of connective tissue remodelling. Nat Rev Mol Cell Biol. 2002;3:349-63.

25. Ikeda T, Miyata Y, Tsutani Y, Misumi K, Arihiro K, Okada M. Fibrinogen/ thrombin-based collagen fleece (TachoComb(R)) promotes regeneration in pulmonary arterial injury. Eur J Cardiothorac Surg. 2012;41:926-32.

Readers who found these articles interesting may also like to read the following papers found in recent and future issues of our sister publications, Seminars in Thoracic and Cardiovascular Surgery and Operative Techniques in Thoracic and Cardiovascular Surgery!

News and Views: Marc de Perrot. Novel induction therapies for pleural mesothelioma. Semin Thorac Cardiovasc Surg. Autumn 2014;26(3):192-200.

State of the Art: Yolonda Colson. Current Innovations in Sentinel Lymph Node Mapping for the Staging and Treatment of Resectable Lung Cancer. Semin Thorac Cardiovasc Surg. Autumn 2014;26(3):201-209.

State of the Art: Prasad Adusumilli. The IASLC/ATS/ERS Lung Adenocarcinoma Classification: What the surgeon should know. Semin Thorac Cardiovasc Surg. Autumn 2014;26(3):210-222.

Current Readings: Robert Suh. Percutaneous Ablation for Pulmonary Metastatic Disease. Semin Thorac Cardiovasc Surg. Autumn 2014;26(3):239-248.

Current Readings: Anne Tsao. Window-of-Opportunity Trials for Thoracic Malignancies. Semin Thorac Cardiovasc Surg. Expected publication April 2015.

State of the Art: Isabelle Schmitt-Opitz. Induction Therapy for Mesothelioma. Semin Thorac Cardiovasc Surg. Expected publication August 2015.

State of the Art: David Schrump. Precision Therapy for Lung Cancer: Tyrosine Kinase Inhibitors and Beyond. Semin Thorac Cardiovasc Surg. Expected publication August 2015. 